\title{
Correlação entre grau de conversão, microdureza e conteúdo inorgânico em compósitos
}

\section{Correlation between degree of conversion, microhardness and inorganic content in composites}

\author{
Alisson Discacciati Neves* \\ José Augusto César Discacciati** \\ Rodrigo Lambert Oréfice*** \\ Wellington Corrêa Jansen****
}

\begin{abstract}
RESUMO: Este estudo teve como objetivo avaliar a correlação entre grau de conversão e microdureza em resinas compostas, e o efeito do conteúdo de partículas e do tipo de unidade fotoativadora sobre esses parâmetros. Três resinas compostas (Artglass ${ }^{\circledR}$, Solidex ${ }^{\circledR}$ e Zeta LC ${ }^{\circledR}$ ) foram polimerizadas em três diferentes unidades laboratoriais (UniXS ${ }^{\circledR}$, Solidilite ${ }^{\circledR}$ e unidade Experimental). Para cada material, quinze corpos-de-prova foram confeccionados em uma matriz metálica, e submetidos às análises do grau de conversão, através de espectroscopia de infravermelho, e da microdureza. O conteúdo de partículas inorgânicas foi determinado por análise termogravimétrica (TGA). O comportamento conjunto das variáveis - grau de conversão e microdureza - foi medido através do coeficiente de correlação de Pearson. Para a resina Artglass $^{\circledR}$, o grau de conversão variou de $37,5 \%$ a $79,2 \%$, com valores de microdureza de 32,4 a $50,3(r=0,904)$. Para a resina Solidex ${ }^{\circledR}$, o grau de conversão variou de $41,2 \%$ a $60,4 \%$, com valores de microdureza de 33,3 a 44,1 ( $\left.\mathrm{r}=0,707\right)$. Para a resina Zeta $L^{\circledR}{ }^{\circledR}$, os valores de conversão e microdureza foram, respectivamente, de $62,0 \%$ a $78,0 \%$ e de 22,6 a 33,6 ( $\mathrm{r}=0,710)$. Concluiu-se que o uso das diferentes unidades resultou em variações dos valores de conversão em função das características específicas de cada unidade. Para cada material, uma forte correlação entre conversão e microdureza foi observada. Além disso, quando materiais diferentes foram comparados, observou-se que o conteúdo de partículas inorgânicas afetou diretamente os valores de microdureza, não interferindo no grau de conversão.
\end{abstract}

DESCRITORES: Resinas compostas; Partículas inorgânicas.

\begin{abstract}
The purpose of this study was to evaluate the correlation between degree of conversion and microhardness in dental composites, as well as the effect of the inorganic content and type of photo-curing unit on these parameters. Three indirect composites (Artglass ${ }^{\mathrm{TM}}$, Solidex ${ }^{\mathrm{TM}}$ and Zeta $\mathrm{LC}^{\mathrm{TM}}$ ) were polymerized by means of three different laboratorial units (UniXS ${ }^{\mathrm{TM}}$, Solidilite ${ }^{\mathrm{TM}}$ and an experimental device). For each material, fifteen samples were prepared using a metal matrix. The degree of conversion was analyzed by means of infrared spectroscopy, and microhardness was also assessed. The inorganic content was measured by means of thermogravimetric analysis (TGA). The Pearson's test was carried out in order to determine correlations. The degree of conversion of Artglass ${ }^{\mathrm{TM}}$ ranged from $37.5 \%$ to $79.2 \%$, and its microhardness, from 32.4 to $50.3(\mathrm{r}=0.904)$. The degree of conversion of Solidex ${ }^{\mathrm{TM}}$ ranged from $41.2 \%$ to $60.4 \%$, and its microhardness, from 33.3 to $44.1(\mathrm{r}=0.707)$. The degree of conversion and the microhardness of Zeta LC ${ }^{\mathrm{TM}}$ ranged from $62.0 \%$ to $78.0 \%$ and from 22.6 to 33.6 , respectively $(\mathrm{r}=0.710)$. It was concluded that the utilization of different photo-curing units caused variations on the degree of conversion, as a result of specific characteristics of each unit. For each material, there was strong correlation between the degree of conversion and microhardness. In addition, when different materials were compared, microhardness was more affected by filler content than by the degree of conversion. DESCRIPTORS: Composite resins; Inorganic particles.
\end{abstract}

\section{INTRODUÇÃO}

As resinas compostas constituem hoje o material de escolha para a restauração estética dos elementos dentais. As primeiras formulações desses materiais demonstraram, com o tempo, baixa resistência ao desgaste, em função do tamanho e formato das partículas de carga. Modificações em sua composição resultaram em melhorias significativas das propriedades, possibilitando a ampliação das indicações dos sistemas resinosos.

Nestes materiais, o grau de conversão dos monômeros apresenta influência sobre sua estabilidade química. A presença de duplas ligações carbônicas não convertidas poderia tornar o ma-

\footnotetext{
*Mestre em Odontologia, Área de Materiais Dentários; **Professor Assistente do Departamento de Odontologia Restauradora; ***Professor Adjunto do Departamento de Engenharia Metalúrgica e de Materiais; ***Professor Titular do Departamento de Odontologia Restauradora - Universidade Federal de Minas Gerais.
} 
Neves AD, Discacciati JAC, Oréfice RL, Jansen WC. Correlação entre grau de conversão, microdureza e conteúdo inorgânico em compósitos. Pesqui Odontol Bras 2002;16(4):349-354.

terial mais susceptivel a reações de degradação, resultando em redução da estabilidade de cor e na liberação de subprodutos como formaldeído e ácido metacrílico ${ }^{5,10,19}$. Além disso, avaliações clínicas e laboratoriais têm evidenciado a relação entre o grau de conversão e as propriedades do material $^{3,13,17}$. Por outro lado, o efeito da conversão sobre estas propriedades pode ser dissimulado devido a outros fatores, como a concentração de partículas de carga.

O objetivo deste trabalho foi avaliar a correlação entre grau de conversão e microdureza em resinas compostas indiretas, e o efeito do conteúdo de partículas inorgânicas e do tipo de unidade fotoativadora sobre esses parâmetros.

\section{MATERIAL E MÉTODOS}

Três resinas compostas, indicadas para técnica indireta, foram avaliadas: Artglass ${ }^{\circledR}$ (DA3, lote 040110, Heraeus Kulzer GmbH, Wehrheim, Alemanha), Solidex ${ }^{\circledR}$ (A3B, lote 109975, Shofu Inc., Kyoto, Japão) e Zeta LC $^{\circledast}$ (A3, lote $5726 J$, Vita Zahnfabrik GmbH, Bad Säckingen, Alemanha).

Para as análises de grau de conversão e microdureza, corpos-de-prova (CP) de $15 \mathrm{~mm}$ de diâmetro e $1 \mathrm{~mm}$ de espessura foram confeccionados em uma matriz metálica. O material foi inserido na matriz, sobre uma lâmina de vidro; uma segunda lâmina foi posicionada sobre o material, sobre a qual foi aplicada uma carga de 0,5 kgf durante $30 \mathrm{~s}$, permitindo a acomodação do material. A utilização das lâminas de vidro teve como objetivos produzir uma superfície lisa e plana, e prevenir a formação da camada de dispersão.

Três unidades de fotoativação laboratoriais foram utilizadas em combinação com os materiais: unidade UniXS ${ }^{\circledast}$ (Heraeus Kulzer GmbH, Wehrheim, Alemanha); unidade Solidilite ${ }^{\circledast}$ (Shofu Inc., Quioto, Japão); e uma unidade de fotoativação Experimental, desenvolvida no Departamento de Odontologia Restauradora da UFMG. As informações relativas às unidades são sumarizadas na Tabela 1 .

Para cada resina, quinze CP foram obtidos, divididos em três grupos de acordo com a unidade de luz utilizada. No aparelho UniXS ${ }^{\circledR}$, os CP foram submetidos a um tempo total de ativação de 270 s; para os aparelhos Solidilite ${ }^{\circledR}$ e Experimental, o tempo total de exposição foi de $240 \mathrm{~s}$.

Ao término da fotoativação, cada $\mathrm{CP}$ foi removido da matriz, e armazenado em ambiente seco e escuro a $37^{\circ} \mathrm{C}$, por $24 \mathrm{~h}$.
TABELA 1 - Unidades de fotoativação utilizadas.

\begin{tabular}{l|c|c|c}
\hline \hline \multicolumn{1}{c|}{ Unidade } & Voltagem & Fonte de luz & $\begin{array}{c}\text { Comprimento de } \\
\text { onda da radiação }\end{array}$ \\
\hline UniXS $^{\circledR}$ & $220 \mathrm{~V}$ & $\begin{array}{c}\text { Xenônio } \\
\text { (2 lâmpadas) }\end{array}$ & $320-520 \mathrm{~nm}$ \\
\hline Solidilite $^{\circledR}$ & $110 \mathrm{~V}$ & $\begin{array}{c}\text { Halógena, } \\
150 \mathrm{~W} \\
(4 \text { lâmpadas })\end{array}$ & $400-600 \mathrm{~nm}$ \\
\hline Experimental & $110 \mathrm{~V}$ & $\begin{array}{c}\text { Halógena, } \\
300 \mathrm{~W}\end{array}$ & - \\
\hline \hline
\end{tabular}

\section{Determinação do grau de conversão}

O grau de conversão dos monômeros foi obtido através de espectroscopia de infravermelho (FTIR, Perkin Elmer 1760X, Norwalk, EUA), utilizando o método da Reflexão Total Atenuada (ATR).

O percentual de duplas ligações carbônicas não convertidas $(\% \mathrm{C}=\mathrm{C})$ foi determinado pela taxa da intensidade de absorção entre ligações $\mathrm{C}=\mathrm{C}$ $\left(1.638 \mathrm{~cm}^{-1}\right)$ e ligações $\mathrm{C}=\mathrm{O}\left(1.730 \mathrm{~cm}^{-1}\right)$, antes e após a polimerização. O grau de conversão (GC) correspondente foi calculado pela subtração desta taxa de $100 \%$.

$$
\begin{aligned}
& (\% C=C)=\frac{(A b s[C=C] / \text { Abs }[C=O]) \text { polimero }}{(A b s[C=C] / \text { Abs }[C=O]) \text { monômero }} 2100 \% \\
& G C=100 \%-(\% C=C)
\end{aligned}
$$

\section{Determinação da microdureza}

Após a análise no infravermelho, os $\mathrm{CP}$ foram submetidos a ensaio de microdureza (Microhardness Tester FM, Future Tech Corp., Tóquio, Japão), utilizando uma ponta Vickers, com carga de $0,2 \mathrm{kgf}$ aplicada durante $15 \mathrm{~s}$. Para cada superficie (superior e inferior) dos $\mathrm{CP}$, um valor médio de microdureza foi obtido a partir de seis endentações.

\section{Determinação do conteúdo inorgânico}

O conteúdo de partículas inorgânicas de cada resina foi determinado através de análise termogravimétrica (TGA), utilizando fragmentos dos CP aquecidos à temperatura de $800^{\circ} \mathrm{C}$.

\section{Tratamento estatístico}

Para todos os grupos, valores de média e desvio-padrão (DP) relativos ao grau de conversão e à microdureza foram obtidos a partir de cinco CP. Os resultados de conversão foram comparados através de análise de variância (ANOVA) a três fatores e teste de Duncan, ao nível de significância de 5\%. 
Neves AD, Discacciati JAC, Oréfice RL, Jansen WC. Correlação entre grau de conversão, microdureza e conteúdo inorgânico em compósitos. Pesqui Odontol Bras 2002;16(4):349-354.

O comportamento conjunto das variáveis grau de conversão e microdureza foi observado por meio de diagramas de dispersão, e medido através do coeficiente de correlação de Pearson (r), ao nível de significância de $5 \%$. A variação do grau de conversão em função da microdureza foi determinada através da regressão linear simples.

\section{RESULTADOS E DISCUSSÃO}

Os resultados do grau de conversão são apresentados na Tabela 2 . A análise de variância indicou que a conversão foi influenciada pelo tipo de material $(F=119,79 ; p=0,00)$, unidade de fotoativação $(\mathrm{F}=13,75 ; \mathrm{p}=0,00)$ e superfície do $\mathrm{CP}$ $(F=87,76 ; p=0,00)$. Além disso, as interações material-unidade $(\mathrm{F}=12,03 ; \mathrm{p}=0,00)$, material-superfície $(F=18,05 ; p=0,00)$, unidade-superfície $(F=12,03 ; p=0,00)$ e a interação entre os três fatores $(F=9,55 ; p=0,00)$ foram significantes.

Observou-se que diferentes valores de conversão foram obtidos para os materiais, o que pode ser atribuído às variações de composição, incluindo sistemas monoméricos, concentração de diluentes, tipo e quantidade de partículas, e concentração de iniciadores.

Para cada material, o uso das diferentes unidades de luz resultou em variações nos valores de conversão, as quais podem ser explicadas pelas di- ferenças nas características de cada unidade, como tipo, número e disposição das lâmpadas, potência e calor gerado no interior dos aparelhos. Variações significativas da performance de polimerização foram também observadas em outros estudos ${ }^{4,6,7,12,15,16}$, através de análises do grau de conversão, da profundidade de polimerização e das propriedades físicas de resinas polimerizadas por diferentes unidades. Resultados insatisfatórios com relação à efetividade da polimerização podem ocorrer devido a uma maior distância entre fonte de luz e material, a um inadequado tempo de exposição, e a baixas intensidades da luz emitida ${ }^{8,9,11}$.

Com relação aos resultados da microdureza (Tabela 3), pode-se observar, de forma similar aos resultados de conversão, que os valores obtidos variaram entre os materiais. A resina Artglass ${ }^{\circledast}$ apresentou os maiores valores de microdureza, enquanto a resina Zeta $\mathrm{LC}^{\circledast}$ demonstrou os menores valores; resultados intermediários foram obtidos para a resina Solidex ${ }^{\circledast}$.

Uma relação direta entre o conteúdo de partículas e a microdureza pode ser observada, uma vez que as resinas Artglass ${ }^{\circledR}$, Solidex ${ }^{\circledR}$ e Zeta LC $^{\circledast}$ apresentam percentuais médios de partículas inorgânicas de aproximadamente $67 \%$, 55\% e $44 \%$ em peso, respectivamente, conforme os dados obtidos na análise termogravimétrica (Gráfico 1). Correlações significativas

TABELA 2 - Médias e desvio padrão do grau de conversão (em \%).

\begin{tabular}{l|c|c|c|c}
\hline \hline \multirow{2}{*}{ Unidade } & \multirow{2}{*}{ Superfície } & \multicolumn{3}{|c}{ Resinas } \\
\cline { 2 - 5 } & & Artglass $^{\circledR}$ & Solidex $^{\circledR}$ & Zeta LC $^{\circledR}$ \\
\hline \multirow{2}{*}{ UniXS $^{\circledR}$} & Superior & $63,9(2,8)^{\mathrm{de}}$ & $47,9(1,9)^{\mathrm{gh}}$ & $63,1(5,1)^{\mathrm{de}}$ \\
\cline { 2 - 5 } & Inferior & $59,1(0,9)^{\mathrm{ef}}$ & $41,2(4,8)^{\mathrm{hi}}$ & $62,0(2,2)^{\mathrm{de}}$ \\
\hline \multirow{2}{*}{ Solidilite $^{\circledR}$} & Superior & $73,2(4,8)^{\mathrm{abc}}$ & $54,3(4,5)^{\mathrm{fg}}$ & $66,8(2,9)^{\mathrm{bcd}}$ \\
\cline { 2 - 5 } & Inferior & $67,2(3,8)^{\mathrm{bcd}}$ & $46,3(3,1)^{\mathrm{h}}$ & $66,6(4,5)^{\mathrm{cd}}$ \\
\hline \multirow{2}{*}{ Experimental } & Superior & $79,2(2,9)^{\mathrm{a}}$ & $60,4(5,6)^{\mathrm{def}}$ & $78,0(11,2)^{\mathrm{a}}$ \\
\cline { 2 - 5 } & Inferior & $37,5(3,3)^{\mathrm{i}}$ & $42,4(8,8)^{\mathrm{hi}}$ & $73,8(7,1)^{\mathrm{ab}}$ \\
\hline \hline
\end{tabular}

Letras diferentes representam valores estatisticamente diferentes.

TABELA 3 - Médias e desvio padrão da microdureza (VHN).

\begin{tabular}{c|c|c|c|c|c|c}
\hline \hline \multicolumn{1}{c|}{ Unidade } & \multicolumn{2}{|c|}{ UniXS $^{\circledR}$} & \multicolumn{2}{c|}{ Solidilite $^{\circledR}$} & \multicolumn{2}{c}{ Experimental $^{\circledR}$} \\
\hline \multicolumn{1}{c|}{ Superfície } & Superior & Inferior & Superior & Inferior & Superior & Inferior \\
\hline Artglass $^{\circledast}$ & $50,3(1,2)$ & $43,1(1,2)$ & $49,5(1,7)$ & $45,6(0,8)$ & $50,1(0,9)$ & $32,4(0,9)$ \\
\hline Solidex $^{\circledast}$ & $36,7(1,8)$ & $33,3(3,1)$ & $39,3(1,5)$ & $37,3(1,3)$ & $44,1(1,7)$ & $37,6(1,5)$ \\
\hline Zeta LC $^{\circledast}$ & $25,5(0,5)$ & $22,6(0,6)$ & $25,1(0,5)$ & $23,8(0,6)$ & $33,6(1,8)$ & $31,2(1,2)$ \\
\hline \hline
\end{tabular}


Neves AD, Discacciati JAC, Oréfice RL, Jansen WC. Correlação entre grau de conversão, microdureza e conteúdo inorgânico em compósitos. Pesqui Odontol Bras 2002;16(4):349-354.

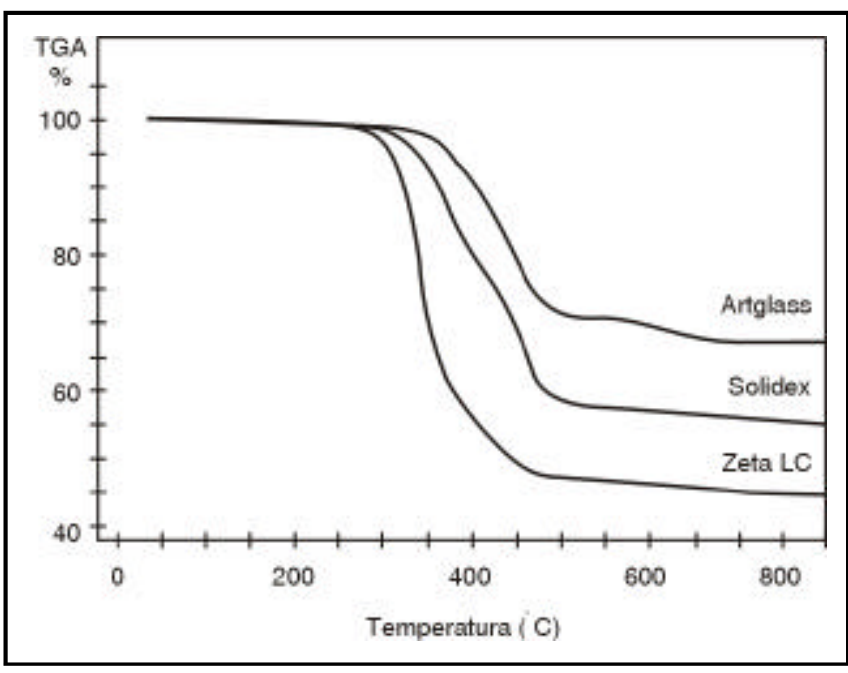

GRÁFICO 1 - Análise termogravimétrica.

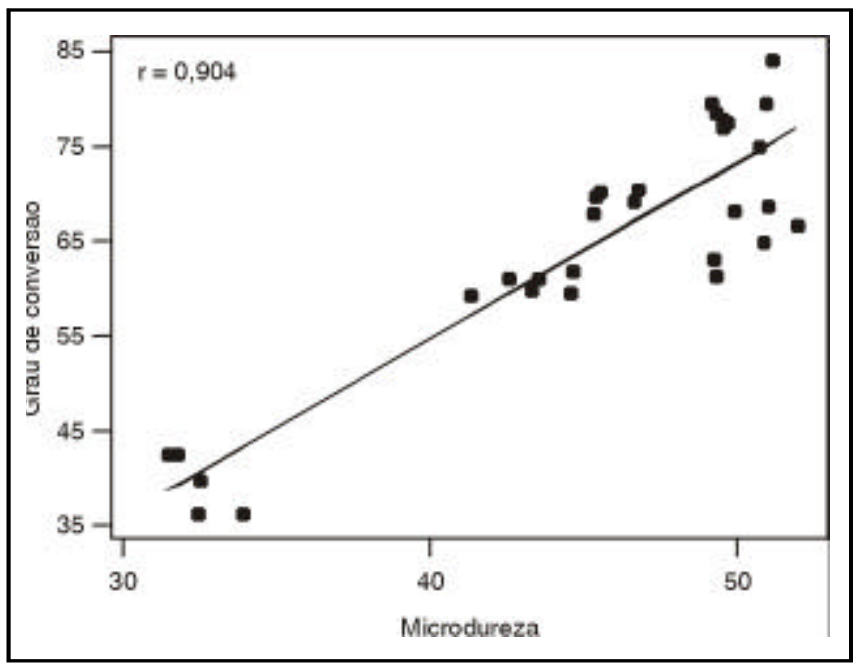

GRÁFICO 2 - Diagrama de dispersão e reta de regressão para a resina Artglass ${ }^{\circledR}(0 \leq \mathrm{r} \leq 0,4$ - correlação fraca; $0,4<\mathrm{r} \leq 0,7$ - correlação moderada; $0,7<\mathrm{r} \leq 1-$ correlação forte).

entre o volume de partículas e as propriedades de resinas compostas foram também relatadas por Chung, Greener ${ }^{1}$ (1990) e Yamaga et al..$^{18}$ (1995). Por outro lado, nenhuma relação pode ser estabelecida entre o grau de conversão e o conteúdo de carga, visto que as resinas Artglass ${ }^{\circledast}$ e Zeta LC $^{\circledR}$ apresentaram valores de conversão similares, apesar das diferenças significativas em relação ao conteúdo inorgânico.

Para a resina Artglass $^{\circledast}$, uma conversão de $73,2 \%$, obtida com o uso do aparelho Solidilite ${ }^{\circledR}$, re-

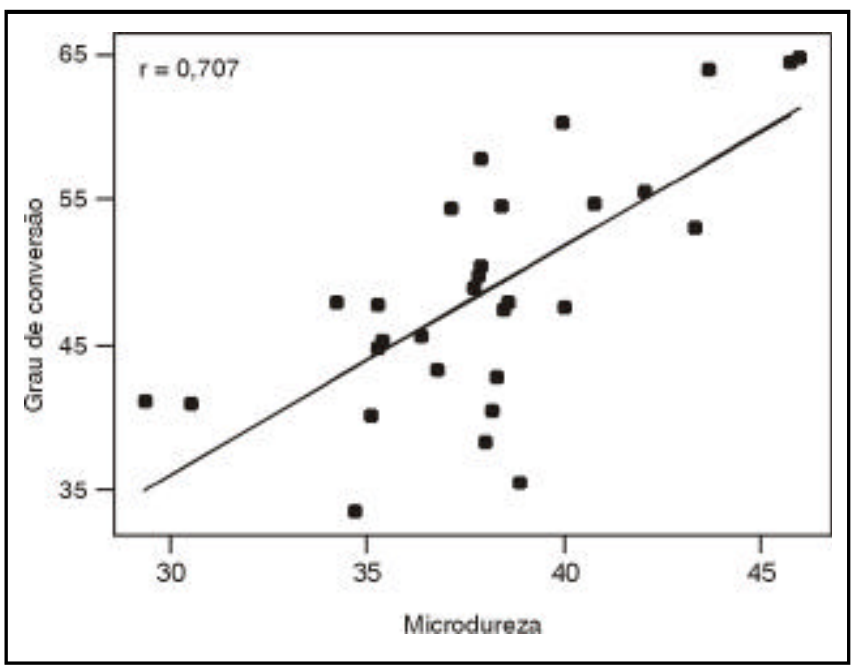

GRÁFICO 3 - Diagrama de dispersão e reta de regressão para a resina Solidex ${ }^{\circledR}(0 \leq \mathrm{r} \leq 0,4$ - correlação fraca; $0,4<\mathrm{r} \leq 0,7$ - correlação moderada; $0,7 \leq \mathrm{r}=1$ correlação forte).

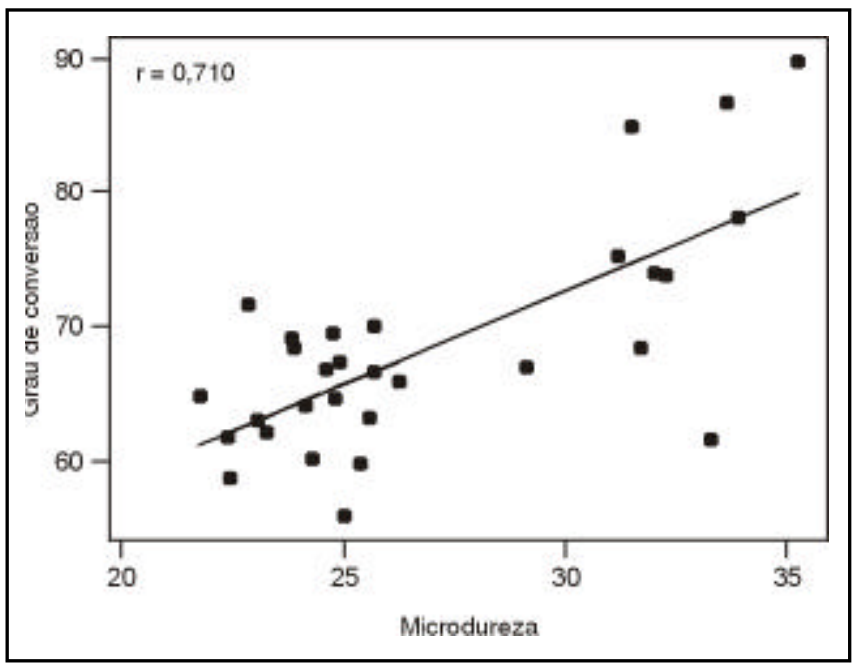

GRÁFICO 4 - Diagrama de dispersão e reta de regressão para a resina Zeta $\mathrm{LC}^{\circledR}(0 \leq \mathrm{r} \leq 0,4$ - correlação fraca; $0,4<\mathrm{r} \leq 0,7$ - correlação moderada; $0,7<\mathrm{r} \leq 1$ correlação forte).

sultou em valores de microdureza de 49,5. Para a resina Solidex ${ }^{\circledR}$, valores de conversão e microdureza de $54,3 \%$ e 39,3 , respectivamente, foram obtidos com o aparelho Solidilite ${ }^{\circledR}$. Para a resina Zeta $\mathrm{LC}^{\circledast}$, o uso desse mesmo aparelho proporcionou valores de conversão de $66,8 \%$ e de microdureza de 25,1 . Pode-se observar que para a resina Solidex ${ }^{\circledast}$, valores de conversão inferiores resultaram em valores de microdureza superiores aos da resina Zeta LC $^{\circledast}$. Estes achados parecem estar de acordo com 
Neves AD, Discacciati JAC, Oréfice RL, Jansen WC. Correlação entre grau de conversão, microdureza e conteúdo inorgânico em compósitos. Pesqui Odontol Bras 2002;16(4):349-354.

Chung, Greener ${ }^{1}$ (1990), que não encontraram correlação entre o grau de conversão e algumas propriedades, entre elas a microdureza, ao analisar diferentes resinas. Entretanto, torna-se difícil estabelecer esse tipo de correlação, uma vez que diferenças causadas pelo grau de conversão podem ser dissimuladas pelo fato de os materiais diferirem em muitos outros aspectos, entre eles o sistema monomérico, o tipo e a quantidade de partículas de carga, e a qualidade da silanização das partículas. Assim, conforme salientado por Ferracane $^{2}$ (1985), a análise de microdureza não pode ser utilizada como método para predizer o grau de conversão quando diferentes materiais são comparados.

Por outro lado, ao contrário de resinas compostas de diferentes sistemas, as propriedades físicas de um dado material estão correlacionadas com o grau de conversão obtido. Essa correlação pode ser identificada através dos diagramas de dispersão referentes às resinas Artglass ${ }^{\circledast}$, Solidex ${ }^{\circledast}$ e Zeta LC ${ }^{\circledast}$, apresentados nos Gráficos 2, 3 e 4 e da aplicação do coeficiente de Pearson, onde os valores obtidos demonstraram uma forte correlação entre grau de conversão e microdureza para os três materiais avaliados. Estes achados corroboram os estudos anteriores de Ferracane ${ }^{2}$ (1985) e Rueggeberg, Craig $^{14}$ (1988), que também observaram uma correlação significativa entre estes parâmetros.

Pela análise da inclinação das retas de regressão apresentadas nos diagramas de dispersão (Gráficos 2, 3 e 4), pode-se observar a influência das variações do grau de conversão sobre a microdureza para cada material. Considerando a resina Artglass ${ }^{\circledR}$, valores de conversão de 63,9\% e 79,2\% resultaram em valores de microdureza de 50,3 e 50,1, respectivamente; para a resina Solidex ${ }^{\circledast}$, valores de conversão de $47,9 \%$ e $54,3 \%$ resultaram, respectivamente, em va-

\section{REFERÊNCIAS}

1. Chung K, Greener EH. Correlation between degree of conversion, filler concentration and mechanical properties of posterior composite resins. $J$ Oral Rehabil 1990; 17(5):487-94.

2. Ferracane JL. Correlation between hardness and degree of conversion during the setting reaction of unfilled dental restorative resins. Dental Mater 1985;1(1):11-4.

3. Ferracane JL, Mitchem JR, Todd R. Wear and marginal breakdown of composites with various degrees of cure. $\mathrm{J}$ Dent Res 1997;76(8):1508-16. lores de microdureza de 36,7 e 39,3 ; para a resina Zeta $\mathrm{LC}^{\circledast}$, resultados de $66,8 \%$ e $78,0 \%$ do grau de conversão proporcionaram valores de microdureza de 25, 1 e 33,6, respectivamente. Assim, as variações da conversão para a resina Zeta $\mathrm{LC}^{\circledR}$ levaram a maiores variações da microdureza, o que é evidenciado pela menor inclinação da reta de regressão obtida para este material. Estes achados sugerem que, para diferentes materiais, variações do grau de conversão podem afetar em maior ou menor extensão suas propriedades físicas.

Essa diferença entre os materiais pode ser explicada, segundo Peutzfeldt, Asmussen ${ }^{13}$ (2000), pelas diferenças no conteúdo de partículas inorgânicas, uma vez que um aumento da conversão poderia não ocasionar melhorias significativas das propriedades físicas para resinas compostas que apresentam altas quantidades de partículas de carga, como a resina Artglass ${ }^{\circledR}$. Contudo, a busca pelos maiores valores possíveis de conversão deveria ainda ser objetivada, uma vez que a presença de monômeros residuais poderia aumentar a susceptibilidade do material à degradação.

\section{CONCLUSÕES}

Considerando os materiais e métodos utilizados, conclui-se que:

1. o uso das diferentes unidades resultou em variações dos valores de conversão em função das características específicas de cada aparelho;

2. para cada material, uma forte correlação entre grau de conversão e microdureza foi observada;

3. quando materiais diferentes foram comparados, observou-se que o conteúdo de partículas inorgânicas afetou diretamente os valores de microdureza, não interferindo no grau de conversão.

\section{AGRADECIMENTOS}

Os autores agradecem à FAPEMIG pelo apoio financeiro (Ref: CDS 678/99).

4. Freiberg RS, Ferracane JL. Evaluation of cure, properties and wear resistance of Artglass dental composite. Am J Dent 1998;11(5):214-8.

5. Imazato S, Tarumi H, Kobayashi K, Hiraguri H, Oda K, Tsuchitani Y. Relationship between the degree of conversion and internal discoloration of light-activated composite. Dental Mater J 1995; 14(1):23-30.

6. Matsumura H, Tanoue N, Atsuda M, Kitasawa S. A metal halide light source for laboratory curing of prosthetic composite materials. J Dent Res 1997;76(2):688-93. 
Neves AD, Discacciati JAC, Oréfice RL, Jansen WC. Correlação entre grau de conversão, microdureza e conteúdo inorgânico em compósitos. Pesqui Odontol Bras 2002;16(4):349-354.

7. Matsumura H, Tanoue N, Atsuda M. Depth of cure of prosthetic composite materials polymerized with laboratory and handheld photo-curing units. J Oral Rehabil 1999; 26(9):698-703.

8. Nomoto R, Uchida K, Hirasawa T. Effect of light intensity on polymerization of light-cured composite resins. Dent Mater J 1994;13(2):198-205.

9. Nomoto R. Effect of light wavelength on polymerization of light-cured resins. Dent Mater J 1997;16(1):60-73.

10. Øyseaed H, Ruyter IE, Sjøvik Kleven IJ. Release of formaldehyde from dental composites. J Dent Res 1998;67(10):1289-94.

11. Pearson GJ, Longman CM. Water sorption and solubility of resin-based materials following inadequate polymerization by a visible-light curing system. J Oral Rehabil 1989;16(1):57-61.

12. Peutzfeldt A. Correlation between recordings obtained with a light-intensity tester and degree of conversion of a light-curing resin. Scand J Dent Res 1994;102(1):73-5.

13. Peutzfeldt A, Asmussen E. The effect of postcuring on quantity of remaining double bonds, mechanical properties, and in vitro wear of two resin composites. J Dent 2000;28(6):447-52.
14. Rueggeberg FA, Craig RG. Correlation of parameters used to estimate monomer conversion in a light-cured composite. J Dent Res 1988;67(6):932-7.

15. Tanoue N, Matsumura H, Atsuta M. Curing depth of a composite veneering material polymerized with seven different laboratory photo-curing units. J Oral Rehabil 1998;25(3):199-203.

16. Tanoue N, Matsumura H, Atsuta M. Properties of four composite veneering materials polymerized with different laboratory photo-curing units. J Oral Rehabil 1998;25(5):358-64.

17. Wendt SL, Leinfelder KF. The clinical evaluation of heat treated composite resin inlays. $J$ Am Dent Assoc 1990;120(2):177-81.

18. Yamaga T, Sato Y, Akagawa Y, Taira M, Wakasa K, Yamaki M. Hardness and fracture toughness of four commercial visible light-cured composite resin veneering materials. J Oral Rehabil 1995;22(12):857-63.

19. Yap AUJ, Lee HK, Sabapathy R. Release of methacrylic acid from dental composites. Dent Mater 2000;16(3):172-9.

Recebido para publicação em 17/10/01

Enviado para reformulação em 20/06/02

Aceito para publicação em 08/08/02 